\title{
The potent cytotoxicity effect of thymoquinone on Chinese ovarian carcinoma cell line HO-8910 mediated by activating autophagy pathway.
}

runzhi zhu ( $\nabla$ runzhizhu1978@163.com )

Jiangsu University Hospital https://orcid.org/0000-0002-9565-7261

Fei Ye

Jiangsu University Hospital

Xitong Wang

Zhejiang University School of Medicine Children's Hospital

Tian Xia

Zhejiang University School of Medicine Children's Hospital

Xiao Yuan

Jiangsu University Hospital

Lanyue Da

Jiangsu University Hospital

Qian Yin

Jiangsu University Hospital

Research

Keywords: thymoquinone, Chinese ovarian cancer, autophagy, HO-8910

Posted Date: January 18th, 2021

DOl: https://doi.org/10.21203/rs.3.rs-145963/v1

License: (9) (i) This work is licensed under a Creative Commons Attribution 4.0 International License.

Read Full License 


\section{Abstract}

Ovarian cancer is known for its highest mortality rate in gynecological malignancy. Previous studies demonstrated that thymoquinone (TQ), a bioactive compound isolated from Nigella sativa L., is a potent anti-ovarian cancer agent, which inhibit tumor growth by modulating apoptotic activity. In this study, we found that the effect of chemotherapy on ovarian cancer cells in vitro varies by race/ethnicity. HO-8910 was established from Chinese patient with ovarian serous carcinoma. The results showed that $\mathrm{HO}-8910$ displayed higher resistance to platinum than SKOV3. And further cell variability assay indicated that TQ exhibited dramatically inhibitory activity on HO-8910 and SKOV3. However, apoptosis and cell cycle analysis suggested TQ had multiple mechanisms for its cytotoxic effect. As for HO-8910, TQ could modulated expression level of several pivotal factors involving autophagy, including PI3K, ATG-7, Bectlin1 and p62, but has little effect on p53, PARP and caspase-3, and no effect on Bax. Our results sustained that TQ was a promising anti-ovarian cancer candidate and its potential application in Chinese patients, even Asian patients, treatment in clinic.

\section{Introduction}

Ovarian carcinoma is a common gynecological malignancy with the highest mortality rate(1). The extremely low 5-year survival rate is contributed by lacking specific symptoms and diagnostic biomarkers in early stages. Actually, more than $70 \%$ patients are in advanced stages (stage III and IV) when diagnosed, which resulting in poor prognosis and high risk in recent $(2,3)$. Ninety percent of ovarian cancers are epithelial, the most common being serous carcinoma, for which incidence and mortality rate varies by race/ethnicity $(2,4)$. For this point of view, it is urgent to investigate potential therapeutic agents, and what is more, race/ethnicity is an important parameter for the therapy of ovarian carcinomas.

Considering that the properties of ovarian cancer in diagnosis, most cases of ovarian cancer are already in different degrees of metastasis (5). Therefore, chemotherapy is the commonly first choice to minimize sizes of tumors for the following surgery and further to provides better prognosis after surgery. Platinumbased chemotherapy is extensively used in the treatment of many advanced ovarian cancer in clinic (6). But nature or acquired drug resistance is common, which is diverse by race with the most possibilities. In our previous studies, we found that the behavior of ovarian cancer cell lines derived from different ethnicities were less in common. Now, the most widely used cell line in ovarian therapy investigation is SKOV3 which is derived from Caucacian. Therefore, we are interesting in ovarian cancer cell lines established from other ethnicities, especially from Chinese, such as HO-8910 (7). Recent studies suggested that thymoquinone (TQ) was a promising anticancer compound which was the key bioactive component of the essential oil from the seeds of Nigella sativa $L$ (8). The cytotoxicity investigations explored the antitumor activity of TQ on several cancer cell lines, such as ovarian, prostate, colon, pancreatic cancer, breast, glioblastoma, leukaemia and so on(8-10). For ovarian cancer, TQ was found to sensitize cisplatin in SKOV3 mediated by Bcl-2 regulated apoptosis (11). In this study, we investigated the effect of TQ both in SKOV3 and HO-8910 compared to cisplatin. And the results indicated unique mechanism of TQ cytotoxicity in HO-8910. 


\section{Materials And Methods}

\subsection{Reagents}

Thymoquinone (TQ) (\#03416) was generously provided by Sigma-Aldrich Chemie GmbH (Taufkirchen, Germany). Primary antibodies against PI3K (\#4292), ATG-7 (\#8558), PARP (\#9542), GAPDH (\#2118), Caspase-3 (\#9665), FASN (\#3180), P62 (8025), Beclin-1 (\#3495), Bax (\#2772) and LC3B (\#3868) were purchased from Cell Signaling Technology (Beverly, MA). Antibody against p53 (DO-l; \#sc-126) was from Santa Cruz Biotechnology. Antibody against Lamp-1 (\#611042) was from BD Transduction Laboratories. Secondary antibodies for immunoblotting were peroxidase goat anti-mouse IgG (\#7076) and peroxidase goat anti-rabbit IgG (\#7074) from Cell Signaling Technology. Secondary antibodies for immunofluorescence staining were Alexa Fluor 594 donkey anti-rabbit (A21207) and Alexa Fluor 488 donkey anti-mouse (A21202) from Thermo Fisher Scientific (Rockford, IL USA). Pefabloc SC was from Roche. Cisplatin injection ( $6 \mathrm{~mL}: 30 \mathrm{mg}$ ) was provided by Hansoh Pharma company.

\subsection{Cell cultures}

Human ovarian serous cystadenocarcinoma HO-8910 (Coriell Cell Repositories) and SKOV3 were obtained from Culture Collection of the Chinese Academy of Sciences (Shanghai, China) where they were characterized by mycoplasma detection, DNA-Fingerprinting, isozyme detection and cell vitality detection. Cells were both cultured in McCoy's 5A Medium (\#PYG0025) from Boster Biological Technology co. Ltd. (China), supplemented with $10 \%$ fetal bovine serum (FBS), $2 \mathrm{mM} \mathrm{L-glutamine} \mathrm{and} 100 \mathrm{unit} / \mathrm{mL}$ penicillin and $100 \mu \mathrm{g} / \mathrm{mL}$ streptomycin. For serum starvation, cells were washed once and incubated overnight in medium containing $0.1 \%$ FBS.

\subsection{Proliferation assay}

HO-8910 and SKOV3 cells were cultured as indicated. Confluent cells were detached with trypsin, seeded into 96 -well plates (8000 cells/well) in 10\% FBS-5A, and allowed to attach for $24 \mathrm{~h}$. After starvation in $0.1 \%$ FBS-5A overnight, cells were treated with indicated doses of test compounds for $24 \mathrm{~h}, 48 \mathrm{~h}$ or $72 \mathrm{~h}$.

The number of viable cells in 96-well plates was determined by the instruction of CellTiter $96 \mathrm{AQ}_{\text {ueous }}$ One Solution Cell proliferation Assay kit (Promega, \#G3580).

\subsection{Cell cycle measurements}

For the cell cycle study, $1 \times 10^{6}$ cells were seeded in 6-well plate and incubated at $37^{\circ} \mathrm{C}$ in $5 \% \mathrm{CO}_{2}$ overnight. The next day cells were starved for $12 \mathrm{~h}$ and then treated with test compounds for $24 \mathrm{~h}$. Cell cycle was determined by flow cytometric analysis using cell cycle detection kit (\#KGA511, KeyGENBioTECH, Nanjing, China) according to the manufacturer's instructions. Briefly, cells were harvested with trypsin and washed twice with PBS. After that, cells were fixed in cold 70\% ethanol and 
stored at $4^{\circ} \mathrm{C}$. On the day of analysis, ethanol was removed by centrifugation and cells were washed twice with PBS, and then treated with RNase: PI mixture for 30 min at $37^{\circ} \mathrm{C}$. The staining samples were processed by a flow cytometer (NovoCyte, ACEA Biosciences, Inc. CA USA). The cell cycle analysis was performed with NovoExpress software (ACEA Biosciences).

\subsection{Detection of cell apoptosis by FACS}

Apoptosis of cells was determined by flow cytometric analysis using Annexin V-kFluor647/PI apoptosis detection kit (\#KGA605, KeyGENBioTECH, Nanjing, China) according to the manufacturer's instructions. After trypsinization, cells were washed twice in cold PBS and suspended at $5 \times 10^{5} / \mathrm{mL}$. Subsequently, 5 $\mu \mathrm{L}$ of Annexin V-kFluor647 reagent and $5 \mu \mathrm{L}$ of PI reagent were added into cell suspension and stored for $15 \mathrm{~min}$ at room temperature in dark place. Apoptotic cells were examined and quantified by using flow cytometry (NovoCyte, ACEA Biosciences, Inc. CA USA).

\subsection{Immunoblotting}

Subconfluent cell cultures were starved and incubated with indicated doses of test compounds for $24 \mathrm{~h}$. Cells were washed once in ice-cold phosphate-buffered saline (PBS) and lysed in RIPA buffer $(0.5 \%$ sodium deoxycholate, $0.1 \%$ sodium dodecyl sulphate (SDS), $1 \%$ Triton X-100, 10\% glycerol, $20 \mathrm{mM}$ Tris$\mathrm{HCl}, \mathrm{pH} 7.4,5 \mathrm{mM}$ EDTA, $150 \mathrm{mM} \mathrm{NaCl}$ ), supplemented with $1 \mathrm{mM}$ Pefabloc and $1 \mathrm{mM}$ sodium orthovanadate. Total cell lysate (TCL) were then clarified by centrifugation at $14000 \times \mathrm{g}$ for $10 \mathrm{~min}$ at 4 ${ }^{\circ} \mathrm{C}$. TCL samples were boiled for $5 \mathrm{~min}$ in SDS sample buffer containing $10 \mathrm{mM}$ dithiothreitol (DTT) and the proteins were separated by SDS-polyacrylamide gel electrophoresis (PAGE) and electro-transferred to PVDF membranes (Immobilon P), which were blocked in 5\% bovine serum albumin (BSA) in $50 \mathrm{mM}$ Tris$\mathrm{HCl}, \mathrm{pH} 7.4,150 \mathrm{mM} \mathrm{NaCl}$, containing $0.1 \%$ Tween 20. Primary antibodies were diluted according to the manufacturer's instructions, or used at $1 \mu \mathrm{g} / \mathrm{ml}$ for home-made antibodies, and incubated with the membranes overnight at $4^{\circ} \mathrm{C}$. After washing, the membranes were incubated for 1 hour with horseradish peroxidase-conjugated anti-rabbit or anti-mouse IgG antibodies (both from Invitrogen Life Technologies), and proteins were visualized using enhanced chemoluminescence (ECL) Western blotting detection system (SuperSignal West Dura Extended Duration Substrate, \#34075) from Thermo Scientific on a cooled charge-coupled (CCD) device camera (Odyssey Fc).

\subsection{Immunofluorescence staining}

The immunofluorescence staining was performed as previously described with modification according to the primary antibody instructions (12). The cells were grown to confluency in 6-well plates onto glass cover slips. The cover slips were washed twice with PBS, prior to fixation with pre-cold methanol for 5 min at room temperature and permeabilized with $0.1 \%$ TritonX-100 (v/v) in PBS for $15 \mathrm{~min}$. After washing three times with PBS, the cover slips were incubated with $2 \%$ BSA (w/v) in PBS for $1 \mathrm{~h}$ to block unspecific 
binding and incubated with primary antibodies $(200 \mathrm{ng} / \mathrm{mL})$ overnight at $4^{\circ} \mathrm{C}$ diluted in PBS containing $0.1 \%$ BSA. They were further washed in PBS and incubated with the secondary antibodies for $1 \mathrm{~h}$ at room temperature. The cover slips were mounted on object slides with Vectashield H-1500 (Vector Laboratories). The cells were photographed under a fluorescence microscope (Axio Imager M2, Zeiss) with an AxioCam MRm digital camera, using a Plan-Apochromat 63 objective lens (Zeiss).

\subsection{Statistical methods}

Data are expressed as mean \pm SD. Statistical significance was determined using the Student's $t$ test. $P<$ 0.05 or $\mathrm{P}<0.01$ was considered statistically significant.

\section{Results}

3.1 The inhibitory effect of thymoquinone on ovarian cancer cells was more efficient than cisplatin

To confirm whether the ethnicity is the key factor to impact the sensitivity of ovarian cancer against chemotherapy, we utilized ovarian cancer cell line HO-8910 and SKOV3 derived from Asian and Caucasian, respectively. Generally, we found that the cytotoxicity of both test compounds, TQ and cisplatin, are in a dose-dependent manner. But it was obvious that cisplatin required longer treatment time to achieve the similar effect of TQ (Figure 1, P < 0.01). Furthermore, we found that HO-8910 cells were less sensitive to the low dose of test compounds compared with SKOV3. As in HO-8910, treatment time of TQ has the least impact on improving the inhibitory effect of TQ on cell proliferation. On the contrary, in SKOV3, both dosage and action time are benefit the effect of these two compounds. It suggested that possibility of race implicated drug effect and resistance in ovarian cancer therapy. Besides, the distinct inhibitory effects of TQ in HO-8910 might be caused by other mechanism which was different from SKOV3.

3.2 The effect of TQ induced apoptosis varies by different origin-derived ovarian cancer cells

To further investigate the role of apoptosis in TQ induced cell death, we compared the apoptosis degree of HO-8910 and SKOV3 by FACS. In general, TQ induced significant apoptosis against ovarian cancer cells compared with cisplatin group (Figure 2A). Interestingly, different origin-derived ovarian cancer cells displayed remarkably diverse sensitivity. For SKOV3, the effect of TQ and cisplatin was quite similar. After treatment, most SKOV3 cells were showed in late apoptotic phase (Q2). However, it was observed that TQ was more efficient on inducing cell death than cisplatin in HO-8910 (Figure 2B, $\mathrm{p}<0.01$ ), which was consistent with the results of our previous assay. Based on the results, HO-8910 seems like to be capable of resistance to cisplatin-induced apoptosis. Additionally, the mechanism of TQ cytotoxicity were more complicated than cisplatin on HO-8910, considering that nearly half of HO-8910 cells showed in nonapoptotic phase (Q1).

It was suggested that TQ could induce HO-8910 death by multiple mechanisms apart from apoptosis. 
3.3 The influence of TQ on cell cycle of ovarian cancer cells by origins.

Given that cell cycle regulation is vital in chemotherapy, we determined the variation of cell cycle profiles (Figure 3A). As noticed before, HO-8910 showed somewhat drug resistance to cisplatin. This might be the reason that there is no significant difference between cisplatin treatment and control groups in HO-8910. But for SKOV3, cisplatin could cause large increasing in S phase, which was consistent with the characteristics of cisplatin acted as a DNA-binding agent. As to TQ, we found that it could increase the percent of G0/G1 phase in HO-8910 but induce dramatically S phase arrest in SKOV3 (Figure 3B, $p<$ 0.05).

To sum up, TQ caused different degree and phase of cell cycle arrest in HO-8910 and SKOV3, which supporting the possibility of varies mechanisms of TQ in HO-8910.

3.4 The marker of autophagy, LC3B, was increased during TQ treatment and showed colocalization with lysosome.

Based on the data, we noticed that the effect of TQ was varies by ovarian cell origins, which, in this case, were Asian and Caucasian. What is more, TQ could induce cell death by another way apart from apoptosis. Given that numerous pieces of evidence indicated autophagic activity of TQ on other cancer cells, we, therefore, investigated this mechanism on HO-8910. The results showed that TQ or nutrients deprivation treatment observably increased the expression of LC3B in cell plasma of HO-8910, which was the typical feature of autophagy $(13,14)$. Additionally, we also found that TQ promoted the colocalization of LC3B with lysosomes under nutrient deprivation, which usually occurred in the mature process of autophagosome $(15,16)$ (Figure 4).

3.5 TQ induced HO-8910 cell death is mainly dependent on autophagy pathway.

In order to identify the signal transduction pathways necessary for TQ-induced HO-8910 cell death we investigated the key factors involved in autophagy and apoptosis pathway. The immunoblotting data indicated that TQ increased PARP cleavage in a dose-dependent manner, but there was slightly increased cleavage of caspase-3 and no effect on Bax expression level. In contrast, we found that TQ affected almost every steps of autophagy pathway including PI3K, Beclin-1, p62 and ATG-7 (14)(Figure 5). TQ could not alter the expression of FASN which was involved in lipid metabolism of tumor cells. Besides, from Immunofluorescence staining results, cytosolic LC3B in HO-8910 was also increased substantially after treated with TQ.

\section{Discussion}

Low drug response and resistance are common in patients with advanced ovarian cancer who is overwhelming majority of ovarian cancer cases $(6,17)$. Therefore, it is necessary to identify novel promising candidate to improve the therapeutic efficacy. Based on the clinical statistics, race could impact the treatment effect of ovarian carcinoma (2). Most studies of candidates screening or 
therapeutic target investigations were based on cell model established from Caucasian, such as SKOV3, Caov-3. However, there are rare reports investigated on ovarian cancer cells derived from other ethnicities, especially Asian. Given that, we are intrigued by the possibility that effects of chemotherapy varies by race. HO-8910 was first established from a Chinese patient with poorly differentiated ovarian serous carcinoma (7). Gene analysis identified HO-8910 as ovarian serous cystadenocarcinoma, the same as SKOV3, which is the majority in ovarian cancer $(18,19)$. In our preliminary screening work, we did find that HO-8910 owned different properties from SKOV3, especially its sensitivity to chemotherapy. Usually, HO-8910 displayed more drug-resistance than SKOV3 to cisplatin which was extensively used in ovarian cancer treatment in clinic. In this study, we found TQ exhibited much better cytotoxicity than cisplatin on HO-8910, although requiring higher dosage to achieve the comparable effect on SKOV3. It is suggested that TQ might be a promising antitumor candidate with broader spectrum in ovarian cancer therapy. Considering that numerous studies demonstrated the apoptotic activity of TQ on SKOV3 $(11,20)$, we further investigate TQ modulation of apoptosis process of HO-8910. Consistent with previous studies, we found that thymoquinone could dramatically induce SKOV3 cell apoptosis. Interestingly, TQ could only induce partial HO-8910 cell apoptosis, and nearly half of cells displayed non-apoptotic death. Then in cell cycle analysis, TQ blocked G1/S phase in HO-8910 other than inducing S phase arrest in SKOV3, which also indicated multiple mechanisms of TQ antitumor activity. Recent evidence showed autophagic activity of TQ on other cancers (21-23). Therefore, we want to confirm whether TQ could modulate HO8910 autophagic process. The results indicated that TQ not only increased the expression of LC3B, but also promoted the colocalization of cytosolic LC3B and lysosomes under the condition of nutrient deprivation in HO-8910. Based on that, we believed TQ could improve the mature of autophagosome under nutrient deficient condition which is common in tumor with rapid growth rate (16). In order to explain the signaling pathway regulated by TQ in HO-8910, we analyzed several pivotal factors involving autophagy process. We found that TQ apparently enhanced the expression level of these factors in almost every step of autophagy process in HO-8910, including PI3K, Beclin-1, ATG-7, p53 and p62. At the same time, TQ could only slightly augment cleavage of PARP and increased Caspase-3 expression level, compared with cisplatin. However, TQ had no effect on Bax expression level, which is different from published work of TQ on SKOV3 (11). Actually, we found that 3-methyladenine (3-MA), the inhibitor of $\mathrm{PI} 3 \mathrm{~K}$ and autophagic pathways, could block the antiproliferative activity of TQ on HO-8910 (data not shown). But Bax inhibitor peptide V5 (BIP-V5) had no effect on TQ cytotoxicity (data not shown) at all, which suggested distinct mechanism of TQ on HO-891.

\section{Conclusion}

In summary, we revealed that TQ could modulate apoptotic and autophagic activities of ovarian cancer cells. But ethnicity of cells had a tremendous impact on the action of chemotherapy. As for ovarian cancer established from the Chinese patient, TQ mainly modulate autophagy process to inhibit tumor proliferation. In this study, we identified TQ as a promising agent with broad spectrum in ovarian cancer therapy. It could be a more potent anti-ovarian candidate than cisplatin in the clinical application of various race patients. 


\section{Abbreviations}

TQ, thymoquinone; Ib, immunoblotting; PBS, phosphate-buffered saline; SDS, sodium dodecyl sulphate; TCL, Total cell lysate; DTT, dithiothreitol; SDS-PAGE, SDS-polyacrylamide gel electrophoresis; BSA, bovine serum albumin; ECL, enhanced chemoluminescence; $C C D$, cooled charge-coupled device.

\section{Declaration}

\subsection{Ethics declarations}

Not applicable.

\subsection{Consent for publication}

Not applicable.

\subsection{Availability of data and materials}

Assure this research's data and materials availability

\subsection{Competing interests}

The authors declare that they have no competing interests.

\subsection{Funds}

This work was supported by grants from affiliated hospital of Jiangsu University funding for talent introduction (JDFYRC2017009; JDFYRC2017011); Jinshan Professor Startup Funding (JSXZ201701). This work was also partially supported by National Natural Science Fundation of China (grant number: 81402189); Natural Science Fundation of Jiangsu Province (grant number: BK20130395); National Natural Science Foundation of China (grant no. 81760359); Jiangsu Overseas Research \& Training Program for University Prominent Young \& Middle-aged Teachers and Presidents.

\subsection{Authors' contributions}

FY and XTW analyzed the data, FY and TX prepared the manuscript, XY囚LD and QY conceived and supervised the study, RZZ revised the manuscript. The author(s) read and approved the final manuscript.

\subsection{Authors' information}

Fei Ye ,xitong Wang and Tian xia equal contribution and co-first authors.

\section{References}


1. Asaduzzaman Khan M, Tania M, Fu S, Fu J. Thymoquinone, as an anticancer molecule: from basic research to clinical investigation. Oncotarget. 2017;8(31):51907-19.

2. Banerjee S, Padhye S, Azmi A, Wang Z, Philip PA, Kucuk O, et al. Review on molecular and therapeutic potential of thymoquinone in cancer. Nutrition and cancer. 2010;62(7):938-46.

3. Bashmail HA, Alamoudi AA, Noorwali A, Hegazy GA, Ajabnoor G, Choudhry H, et al. Thymoquinone synergizes gemcitabine anti-breast cancer activity via modulating its apoptotic and autophagic activities. Scientific reports. 2018;8:11674.

4. Choi KS. Autophagy and cancer. Experimental \& molecular medicine. 2012;44(2):109-20.

5. Chu S-C, Hsieh Y-S, Yu C-C, Lai Y-Y, Chen P-N. Thymoquinone Induces Cell Death in Human Squamous Carcinoma Cells via Caspase Activation-Dependent Apoptosis and LC3-II ActivationDependent Autophagy. PLoS ONE. 2014;9(7):e101579.

6. Ghoneum A, Afify H, Salih Z, Kelly M, Said N. Role of tumor microenvironment in the pathobiology of ovarian cancer: Insights and therapeutic opportunities. Cancer Med. 2018.

7. Levy JMM, Towers CG, Thorburn A. Targeting autophagy in cancer. Nat Rev Cancer. 2017;17(9):52842.

8. Liu X, Dong J, Cai W, Pan Y, Li R, Li B. The Effect of Thymoquinone on Apoptosis of SK-OV-3 Ovarian Cancer Cell by Regulation of Bcl-2 and Bax. International journal of gynecological cancer : official journal of the International Gynecological Cancer Society. 2017;27(8):1596-601.

9. Mou HZ, Xu SH, Zhang YY. The establishment of human ovarian carcinoma cell line HO-8910 and its characteristics. Zhonghua fu chan ke za zhi. 1994;29(3):162-4, 91.

10. Muggia F. Platinum compounds 30 years after the introduction of cisplatin: implications for the treatment of ovarian cancer. Gynecol Oncol. 2009;112(1):275-81.

11. Muinao T, Deka Boruah HP, Pal M. Diagnostic and Prognostic Biomarkers in ovarian cancer and the potential roles of cancer stem cells - An updated review. Experimental Cell Research. 2018;362(1):110.

12. Pazhouhi M, Sariri R, Rabzia A, Khazaei M. Thymoquinone synergistically potentiates temozolomide cytotoxicity through the inhibition of autophagy in U87MG cell line. Iranian Journal of Basic Medical Sciences. 2016;19(8):890-8.

13. Rorsman C, Tsioumpekou M, Heldin C-H, Lennartsson J. The Ubiquitin Ligases c-Cbl and Cbl-b Negatively Regulate Platelet-derived Growth Factor (PDGF) BB-induced Chemotaxis by Affecting PDGF Receptor $\beta$ (PDGFR $\beta$ ) Internalization and Signaling. Journal of Biological Chemistry. 2016;291(22):11608-18.

14. Shen DW, Pouliot LM, Hall MD, Gottesman MM. Cisplatin resistance: a cellular self-defense mechanism resulting from multiple epigenetic and genetic changes. Pharmacol Rev. 2012;64(3):70621.

15. Siegel RL, Miller KD, Jemal A. Cancer statistics, 2018. CA Cancer J Clin. 2018;68(1):7-30. 
16. Taha MM, Sheikh BY, Salim LZ, Mohan S, Khan A, Kamalidehghan B, et al. Thymoquinone induces apoptosis and increase ROS in ovarian cancer cell line. Cellular and molecular biology. 2016;62(6):97-101.

17. Teng Z, Han R, Huang X, Zhou J, Yang J, Luo P, et al. Increase of Incidence and Mortality of Ovarian Cancer during 2003-2012 in Jiangsu Province, China. Frontiers in Public Health. 2016;4(146).

18. Torre LA, Trabert B, DeSantis CE, Miller KD, Samimi G, Runowicz CD, et al. Ovarian cancer statistics, 2018. CA: A Cancer Journal for Clinicians. 2018;68(4):284-96.

19. White $E$. The role for autophagy in cancer. J Clin Invest. 2015;125(1):42-6.

20. Woo CC, Kumar AP, Sethi G, Tan KH. Thymoquinone: potential cure for inflammatory disorders and cancer. Biochem Pharmacol. 2012;83(4):443-51.

21. Xu S, Mou H, Lu G, Zhu C, Yang Z, Gao Y, et al. Gene expression profile differences in high and low metastatic human ovarian cancer cell lines by gene chip. Chin Med J (Engl). 2002;115(1):36-41.

22. Yang ZJ, Chee CE, Huang S, Sinicrope FA. The role of autophagy in cancer: therapeutic implications. Mol Cancer Ther. 2011;10(9):1533-41.

23. Zhu L, Hu Z, Liu J, Gao J, Lin B. Gene expression profile analysis identifies metastasis and chemoresistance-associated genes in epithelial ovarian carcinoma cells. Med Oncol. 2015;32(1):426.

\section{Figures}

A

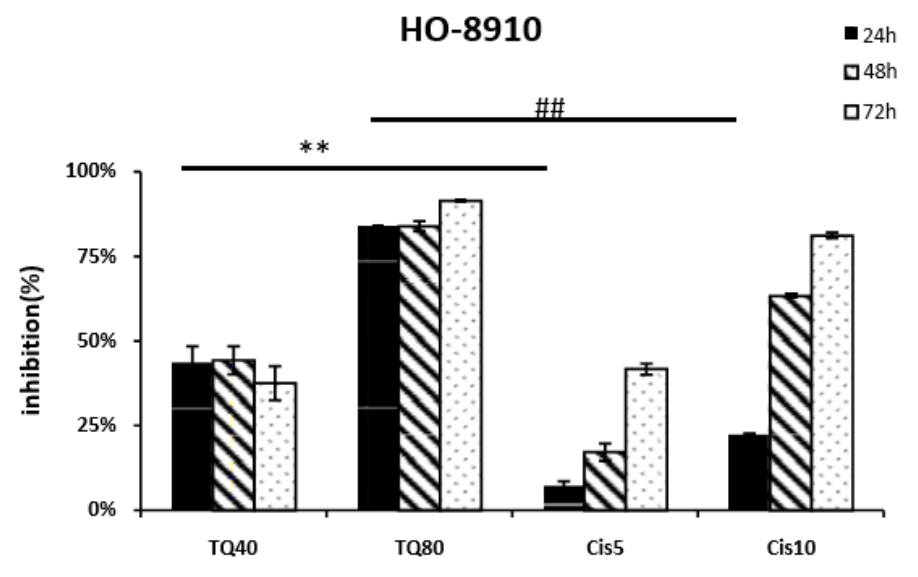

B

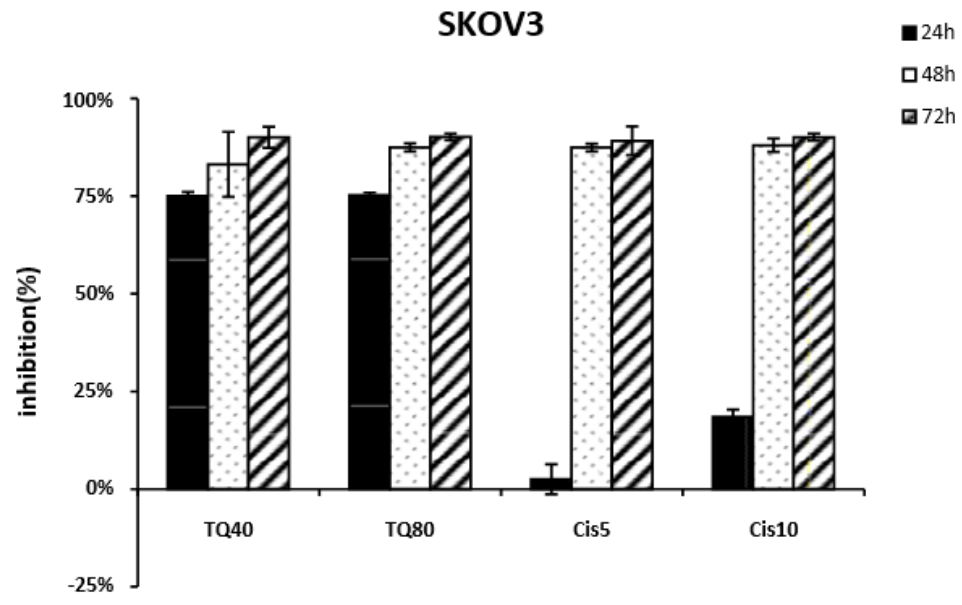

\section{Figure 1}

TQ exhibited potent inhibitory effect on proliferation of human ovarian cancer cell lines HO-8910 and SKOV3. Human ovarian cancer cell lines HO-8910 and SKOV3 were cultured as indications. Cells were seeded in 96-well plates and serum-starved overnight. After $24 \mathrm{~h}, 48 \mathrm{~h}$ and $72 \mathrm{~h}$ treatment, viable cell numbers were determined according to the instruction of CellTiter 96 AQueous One Solution Cell proliferation Assay kit. (A), HO-8910 were treated with indicated doses of TQ or cisplatin. (B), SKOV3 were

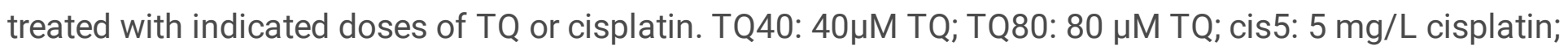


cis 10: $10 \mathrm{mg} / \mathrm{L}$ cisplatin. And the mean values of inhibition rates were present in column graphs $(\mathrm{n}=3$, three independent experiments). ** (TQ40 vs. Cis5 for $24 \mathrm{~h}$ ) or \#\# (TQ80 vs. Cis10 for $24 \mathrm{~h}$ ) indicate $\mathrm{p} \leq$ 0.01 in a Student's t-test.

A

NC

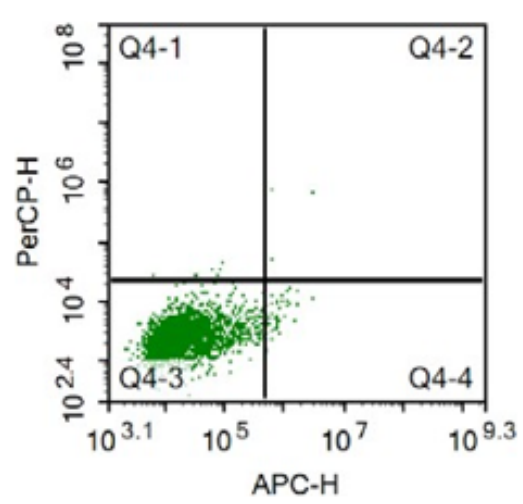

HO-8910

SKOV3

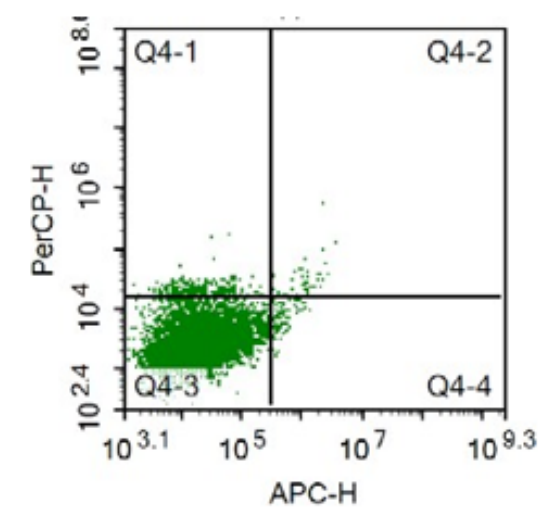

B
Cis $(5 \mathrm{mg} / \mathrm{L})$
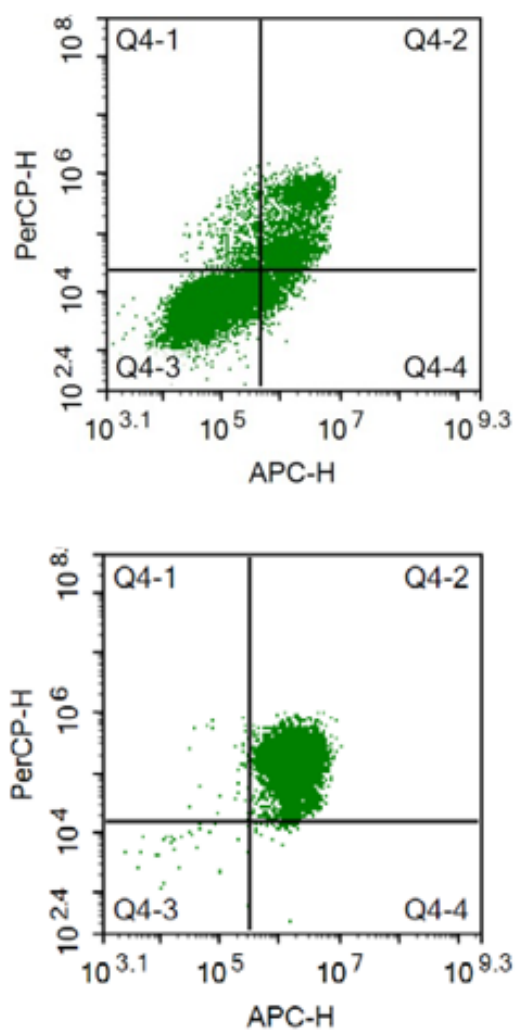

$\mathrm{TQ}(40 \mu \mathrm{M})$
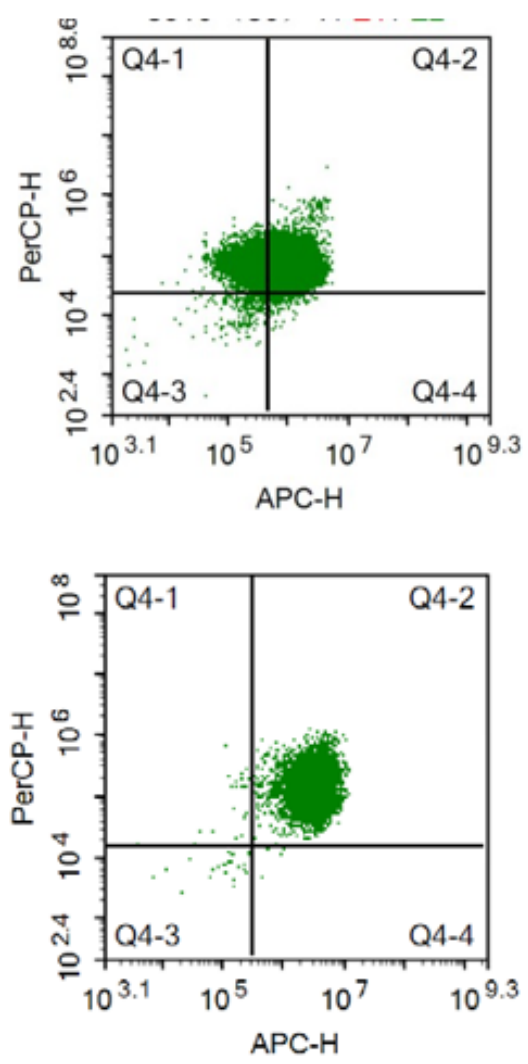

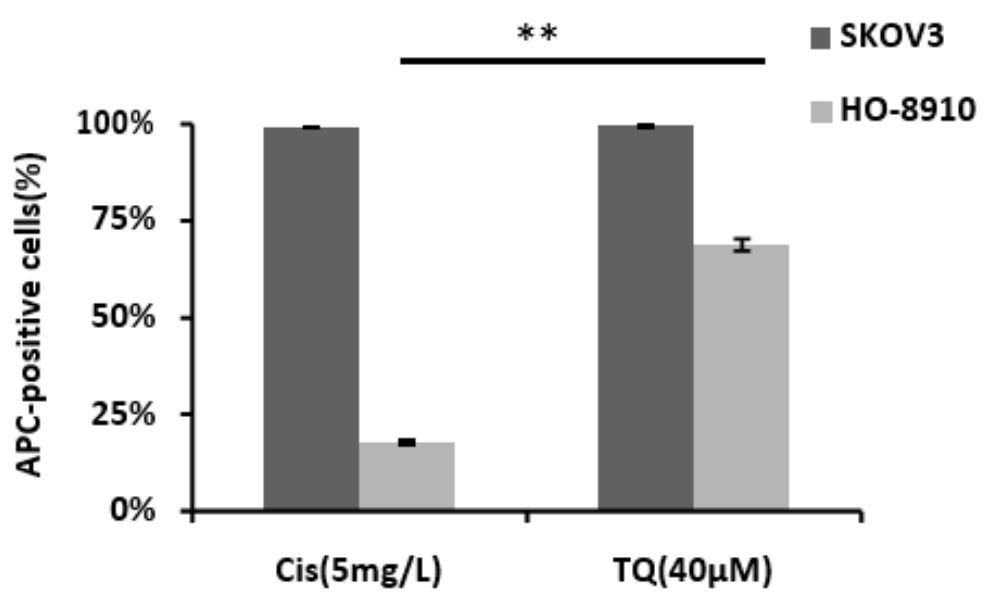

Figure 2

TQ displayed different effects on apoptosis of diverse ethnicity-derived ovarian cancer cells. (A), FACS plots displays differences in cell apoptosis phases of HO-8910 and SKOV3 treated by TQ $(40 \mu \mathrm{M})$ or cisplatin ( $5 \mathrm{mg} / \mathrm{L})$ for $24 \mathrm{~h}$, as determined by FACS analysis. (B), Percentages of cell phases were present 
in column graphs $(n=3$, the statistics analysis was obtained from three independent experiments). ** (TQ40 vs. Cis5 in HO-8910) indicate $p \leq 0.01$ in a Student's t-test.

A
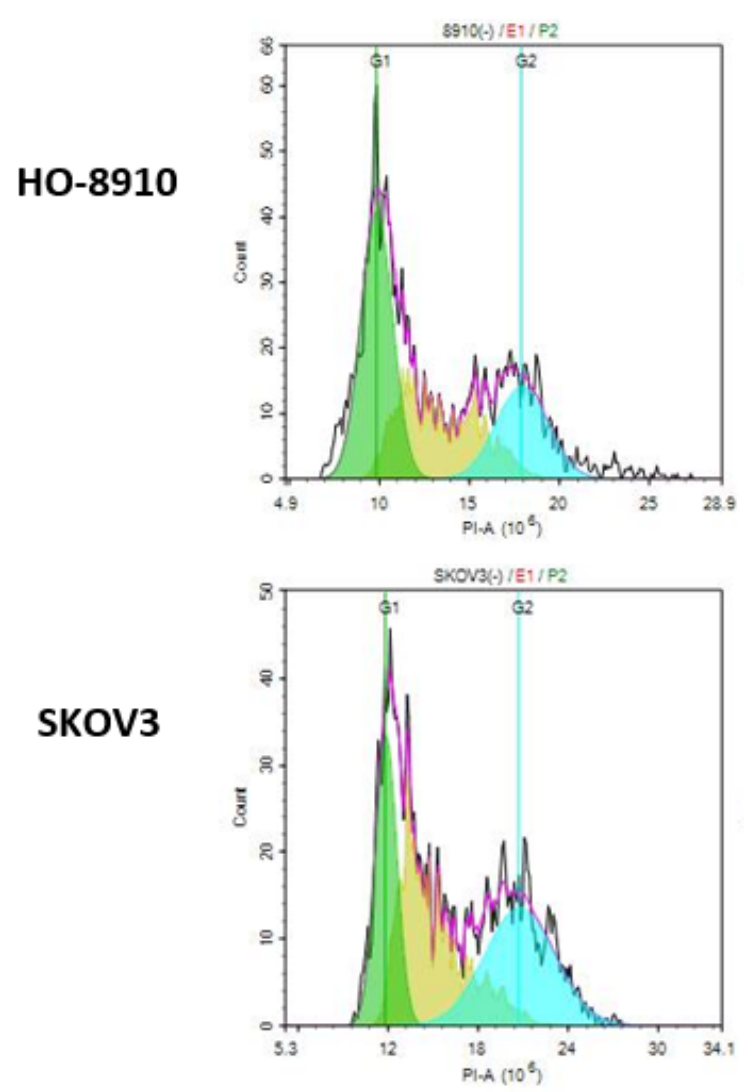

Cis $(5 \mathrm{mg} / \mathrm{L})$
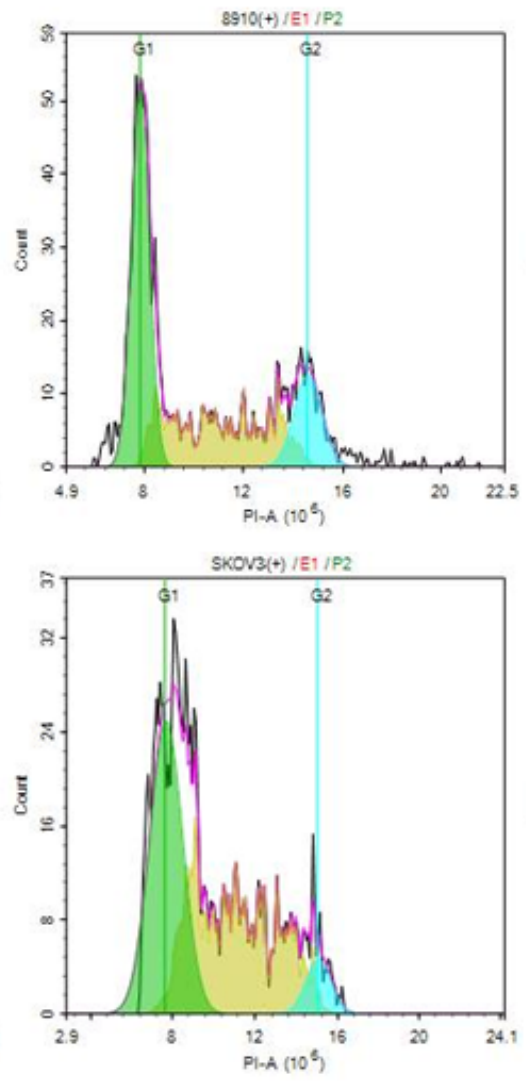

$\mathrm{TQ}(40 \mu \mathrm{M})$
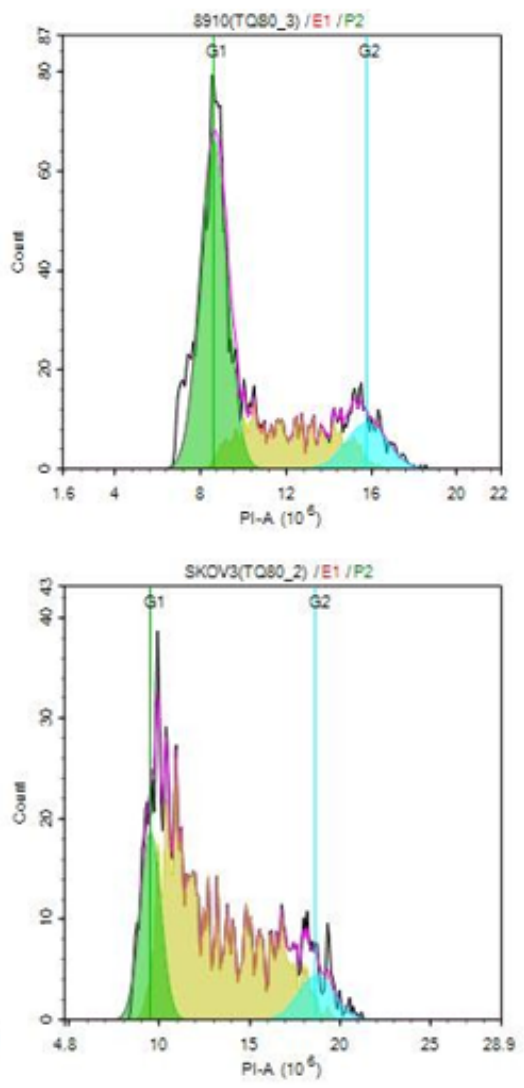

B

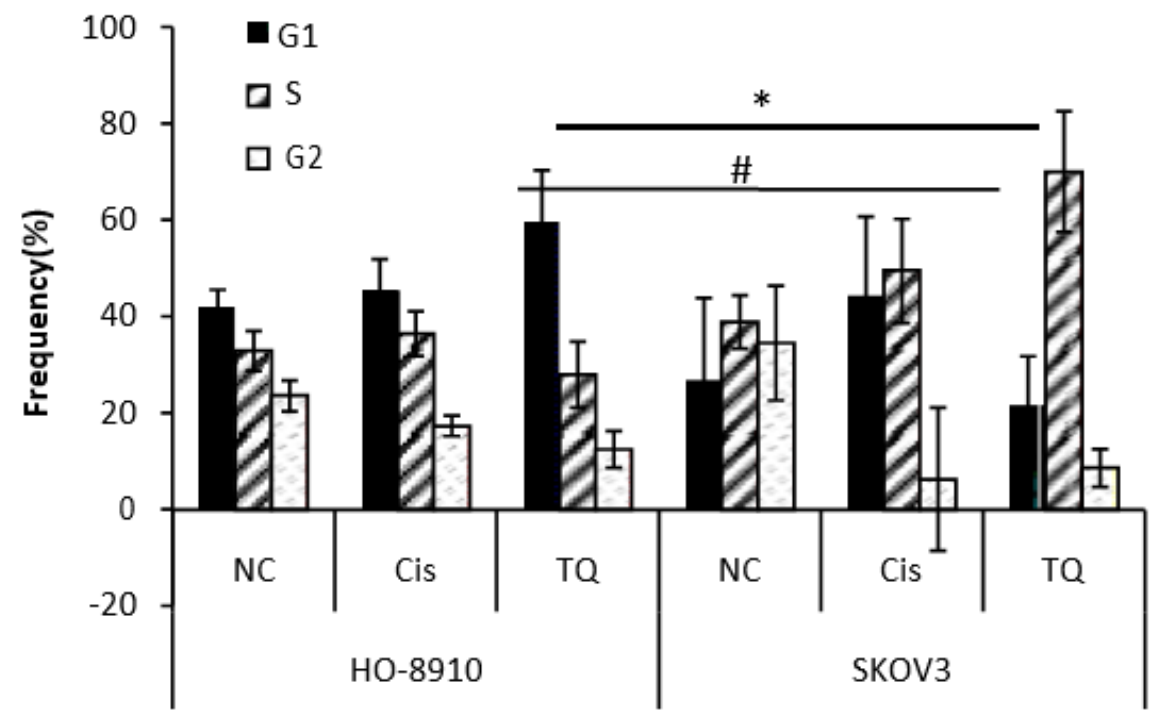

\section{Figure 3}

TQ alters the cell cycle profile of HO-8910, which different from SKOV3. (A), FACS plots displays differences in cell cycle phases of solvent reagent (NC) and test compounds including TQ $(40 \mu \mathrm{M})$ or cisplatin $(5 \mathrm{mg} / \mathrm{L})$ for $24 \mathrm{~h}$, as determined by FACS analysis. (B), Percentages of cell cycle phases were present in column graphs $(n=3)$. HO-8910 treated with TQ have an altered cell cycle profile, with 
significantly less cells with S DNA content $\left(^{*}\right)$, and significantly more with G1-phase DNA content (\#), which was different from SKOV3 (mean \pm SD; * or \# indicate $p \leq 0.05$ in a Student's t-test).

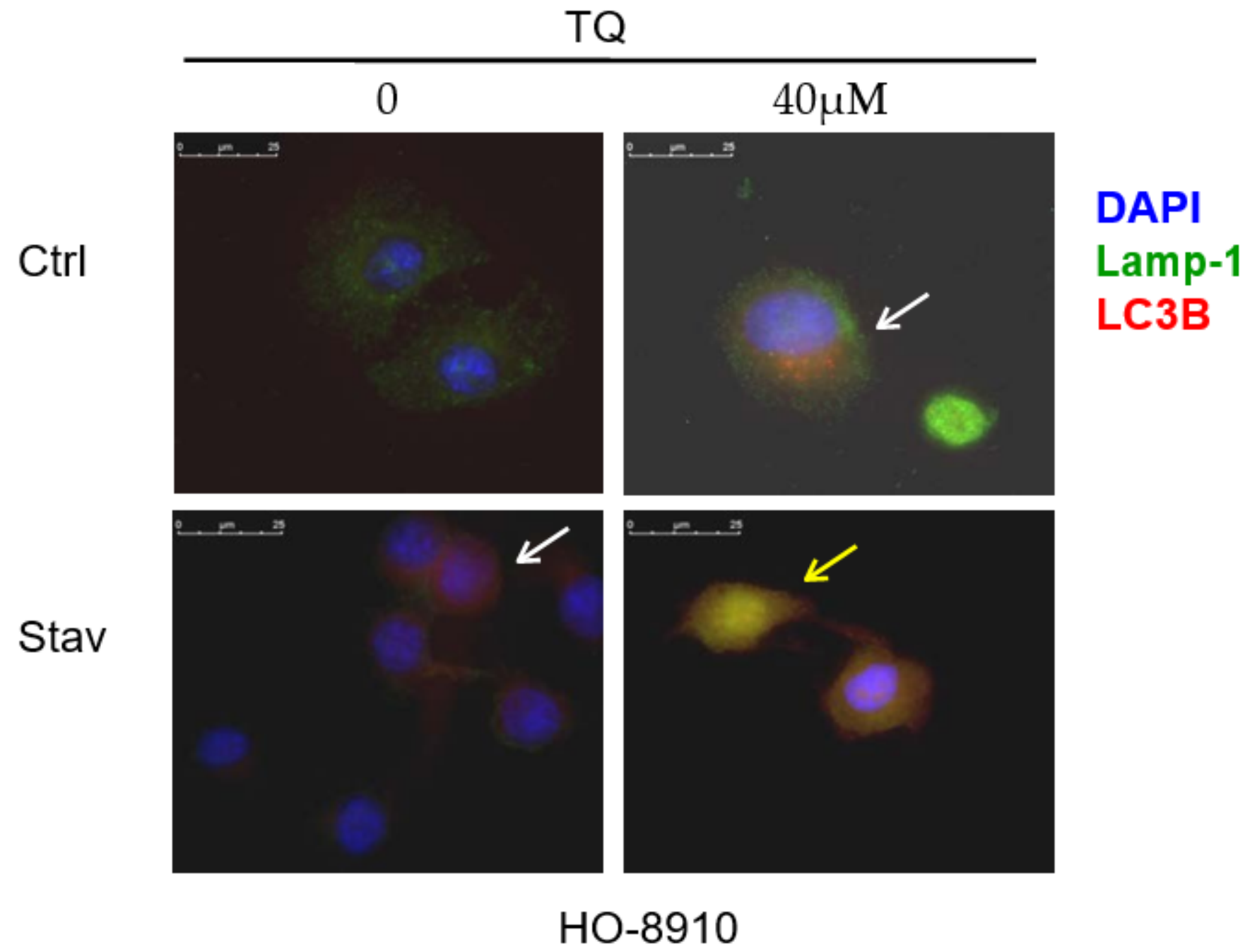

\section{Figure 4}

TQ treatment leads to upregulation and promotes localization of cytosolic LC3B. HO-8910 cells were cultured in growth medium or serum-starved medium overnight and incubated with $40 \mu \mathrm{M}$ TQ for $24 \mathrm{~h}$. The localization of cytosolic LC3B and lysosomes (Lamp-1) in HO-8910 were detected by immunoflurescence staining. Blue: DAPI; Red: LC3B; Green: Lamp-1. LC3B was upregulated significantly after TQ treatment (TQ) or nutrients deprivation (Stav) alone (shown as white arrows), compared with cells in growth medium (Ctrl). TQ could promote colocalization of LC3B and lysosomes under the condition of nutrients deprivation (shown as the yellow arrow). A representative assay of three independent experiments is shown. 


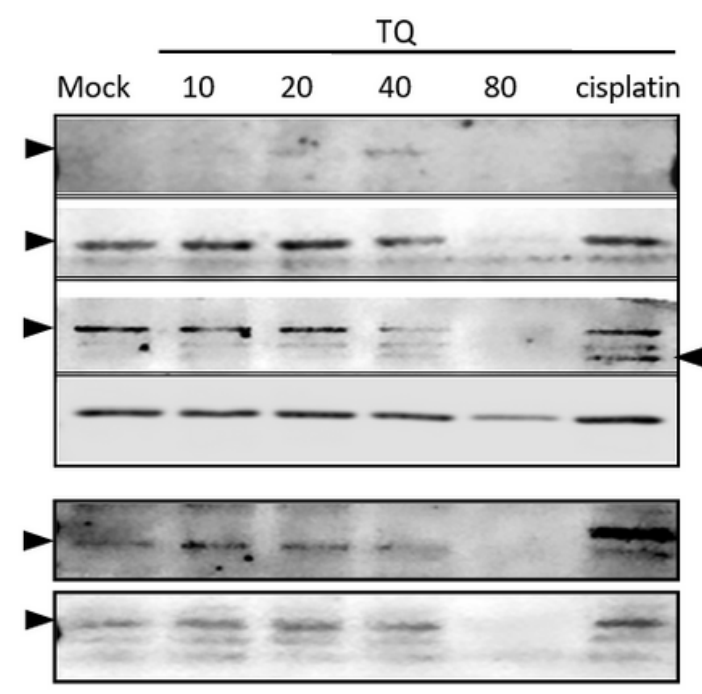

Ib: PI3K

Ib: ATG7

Ib: PARP

Ib: GAPDH

Ib: p53

Ib: Caspase-3

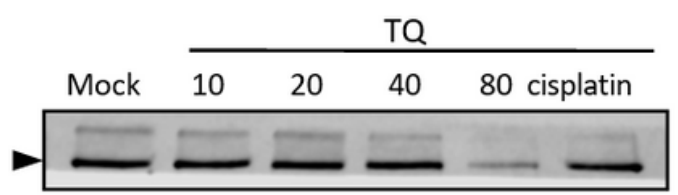

Ib: FASN

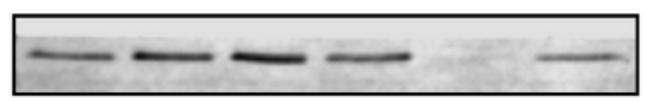

Ib: p62
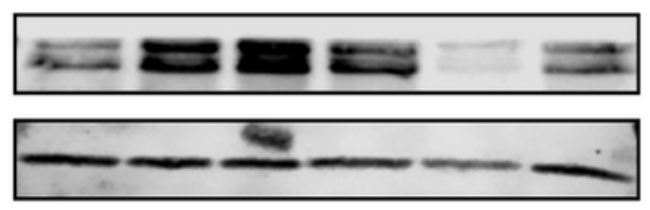

Ib: Beclin-1

Ib: Bax

Ib: GAPDH

\section{Figure 5}

TQ treatment mainly induced expression of key signaling factors in autophagy pathway in HO-8910. HO8910 were treated by series doses of TQ $(10,20,40,80 \mu \mathrm{M})$ or cisplatin $(5 \mathrm{mg} / \mathrm{L})$ for $24 \mathrm{~h}$. Total cell lysates (TCL) were prepared and subjected to SDS-PAGE and protein expression of PI3K, ATG7, p62, Beclin-1, PARP, Caspase-3, p53 and Bax which involved in autophagy or apoptosis process were analyzed by immunoblotting (Ib). GAPDH in HO-8910 cells was detected as loading control by lb. A representative assay of three independent experiments is shown. 